\title{
Laparo-endoscopic single site surgery in pediatrics: Feasibility and surgical outcomes from a preliminary prospective Canadian experience
}

\author{
Aziz Khambati, MD; Elias Wehbi, MD; Walid A. Farhat, MD, FRCSC \\ Division of Urology, Hospital for Sick Children, University of Toronto, Toronto, ON
}

See related article on page 53.

Cite as: Can Urol Assoc J 2015;9(1-2):48-52. http://dx.doi.org/10.5489/cuaj.2379

Published online February 5, 2015.

\section{Abstract}

Introduction: Laparo-endoscopic single-site surgery (LESS) is becoming an alternative to standard laparoscopic surgery. Proposed advantages include enhanced cosmesis and faster recovery. We assessed the early post-operative surgical outcomes of LESS surgery utilizing different instruments in the pediatric urological population in Canada.

Methods: We prospectively captured data on all patients undergoing LESS at our institution between February 2011 and August 2012. This included patient age, operative time, length of stay, complications and short-term surgical outcomes. Different instruments/devices were used to perform the procedures. Access was achieved through a transumbilical incision.

Results: A total of 16 LESS procedures were performed, including seven pyeloplasties, four unilateral and one bilateral varicocelectomies, two simple nephrectomies, one renal cyst decortication and one pyelolithotomy. There was no statistical difference in the operative times, hospital length of stay and cost (pyeloplasty only) in patients undergoing pyeloplasty and varicocelectomy using the LESS technique when compared to an age matched cohort of patients managed with the traditional laparoscopic approach. One pyeloplasty in the LESS group required conversion to open due to a small intra-renal pelvis. There were no immediate or short term post-operative complications; however, one patient experienced a decrease in renal function status post LESS pyeloplasty. Since all procedures were performed by a vastly experienced surgeon at a tertiary center, the generalizability of the results cannot be assessed. Conclusions: There are only a few series that have assessed the role of LESS in pediatric urological surgery. Although our experience is limited by a heterogeneous group of patients with a short follow-up period, the present cohort demonstrates the safety and feasibility of LESS. Further evaluation with randomized studies is required to better assess the role of LESS in pediatric urology.

\section{Introduction}

Surgical techniques and instruments are constantly evolving. The introduction of laparoscopy allowed urologists to minimize patient morbidity. Recently, however, novel modifications to laparoscopy have led to single-port surgery, ${ }^{1-3}$ with laparo-endoscopic single-site surgery (LESS) as an alternative to standard laparoscopic surgery. LESS has been reported in mostly adults, with few pediatric series and no reported cases at Canadian centres. Proposed advantages over laparoscopy include enhanced cosmesis. This is especially true in the pediatric population where multiple incisions may be significant given the relatively smaller size of the patient. Other possible advantages include reduced pain and requirement for analgesics and shorter postoperative stay. ${ }^{4,5}$

We report the first experience with LESS in Canada by describing the feasibility and surgical outcomes after 2 years for different urologic procedures in the pediatric population. We further attempted to evaluate the cost of this technology in comparison to traditional laparoscopy in patients undergoing pyeloplasty.

\section{Methods}

We prospectively captured data on all patients undergoing LESS surgery at a single institution between February 2011 and August 2012. The approval for the introduction of LESS at our institution and the study was obtained from the Departments of Surgery and Bioethics and Quality and Risk Management through a standardized process called "Proposal for Introduction of Innovative Procedures and Devices."6

All surgeries were performed by a single surgeon (WF) through a transumbilical incision under direct vision using an open Hasson technique. The length of the incision varied in size from 1.5 to $2.5 \mathrm{~cm}$, depending on the access device used (Fig. 1a). Procedures performed included dismembered 
pyeloplasty, varicocelectomy, simple nephrectomy, renal cyst decortication, and pyelolithotomy. During the pyeloplasty, a stent was inserted in the traditional laparoscopic manner using an angiocatheter. For the varicocelectomy, the peritoneum was incised to isolate the spermatic veins and en-bloc clipping was performed. In cases where the artery was easily spared, only the veins were clipped. The surgical technique for the pyelolithotomy case has been previously described. ${ }^{7}$ The cases were performed in no specific chronological order.

During our initial experience, several access ports were used. These included the Triport (Olympus/Advanced Surgical Concepts), GelPOINT System (Applied Medical), and the SILS port (Covidien). All working instruments were $5 \mathrm{~mm}$. In the latter cases, typical instruments would include 1 straight and 1 articulating instrument (Covidien) of different lengths, in an attempt to prevent extracorporeal clashing, and an articulating 5-mm camera (Endoeye Flex 5, Olympus) that provides a 100-degree angulation. Insufflation was achieved via the appropriate port channel on the access device, whereby $\mathrm{CO}_{2}$ pneumoperitoneum was achieved and set at $12 \mathrm{mmHg}$ (Fig. 1b). Figure 2 lists the procedure breakdown between different devices.

Patient age, operative time, length of stay, and surgical outcomes were captured. An age-matched historical cohort of traditional laparoscopic dismembered pyeloplasty and varicocelectomy was used for comparative analysis. For the LESS pyeloplasty group, a cost analysis was also performed by accounting for the device type, the operative time, and the length of stay of the patient. The operative time was calculated from the time of the initial skin incision to its closure. Cystoscopy, when required, was also included in the operative time. Two-sample t-test was performed and SPSS v. 21 was used for statistical analysis.

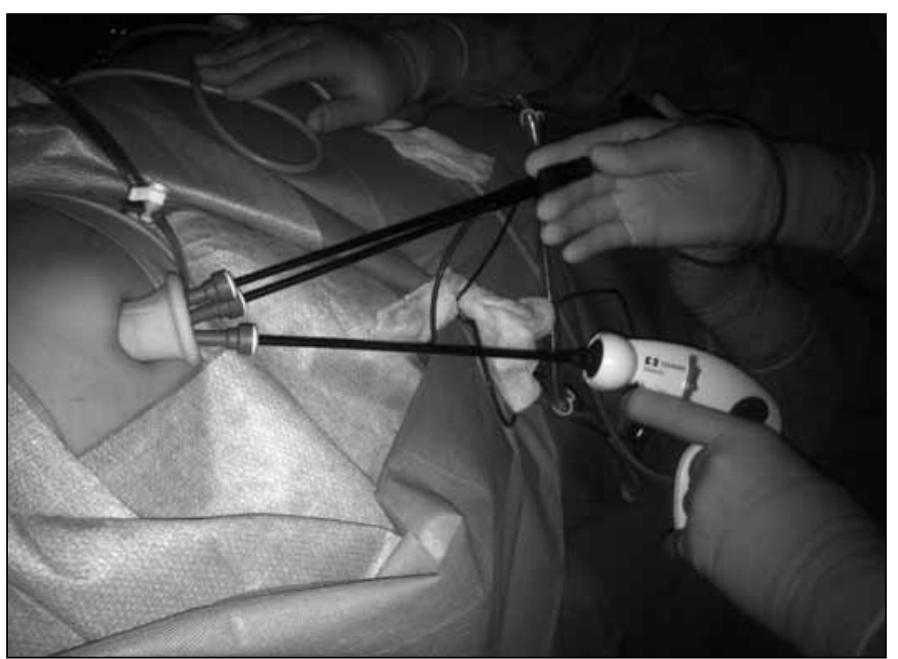

Fig. 1a. Laparo-endoscopic single-site surgery-pyeloplasty using SILS port (Covidien) with Endoeye (Olympus) camera using articulating instruments.

\section{Results}

The 16 LESS surgeries included 7 dismembered pyeloplasties (44\%), 4 unilateral and 1 bilateral varicocelectomies (31\%), 2 simple nephrectomies (13\%), 1 renal cyst decortication $(6 \%)$ in a child with large symptomatic multicystic kidney, and 1 pyelolithotomy (6\%) in a child who had cystinuria and a history or multiple percutaneous nephrolithotomies (Table 1).

A total of 7 patients had LESS pyeloplasties (LESS-P) with mean follow-up of 15.7 months. Patients were matched 1:4 to a similar cohort of patients who underwent traditional laparoscopic pyeloplasties (TL-P). The mean age at surgery was 12.7 years (range: $7.7-17.9$ ) and 12.8 years (range: $7.7-17.8$ ) for LESS-P and TL-P groups, respectively $(p=0.82)$. There was no significant difference in operative times between the 2 groups (LESS-P: 233 minutes [range: 155-250] vs. TL-P: 210 minutes [range: $116-373$ ], $p=0.82$ ). No patients in the TL-P group required conversion to an open procedure, however 1 patient undergoing LESS-P required conversion for failure to progress due to a small intra-renal pelvis. There was no significant difference in length of stay between the groups (LESS-P: 2.2 days [range: 1.53-2.61] vs. TL-P: 2.8 days [1.48-7.6], $p=0.26$ ). The mean cost for LESS-P was \$5088CAD compared to \$5701CAD for TL-P $(p=0.30)$. The non-statistical cost-savings in the LESS-P group was likely driven by the cost accrued by a prolonged hospital stay of 7.6 days in 1 child in the TL-P group. Of the 7 patients who underwent LESS-P, 6 showed improvement in the degree of hydronephrosis. One patient whose ureteropelvic junction (UPJ) obstruction was incidentally diagnosed after trauma developed worsening hydronephrosis and decreasing renal function on the renal scan from $35 \%$ to $5 \%$ at 6 months post-LESS-P. It is unclear whether the deterioration was secondary to the trauma or the sur-

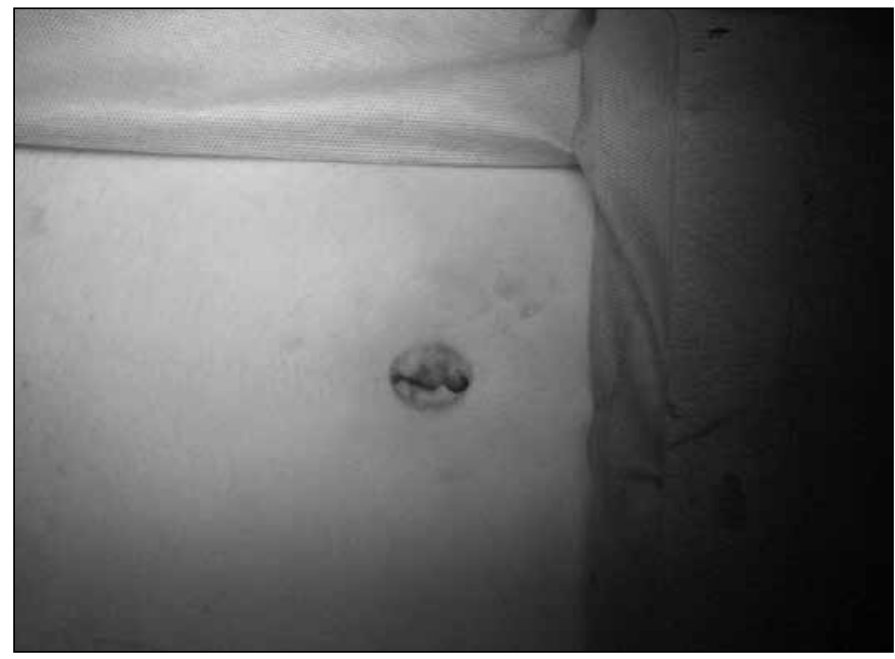

Fig. 1b. Postoperative umbilical incision. 
Khambati et al.

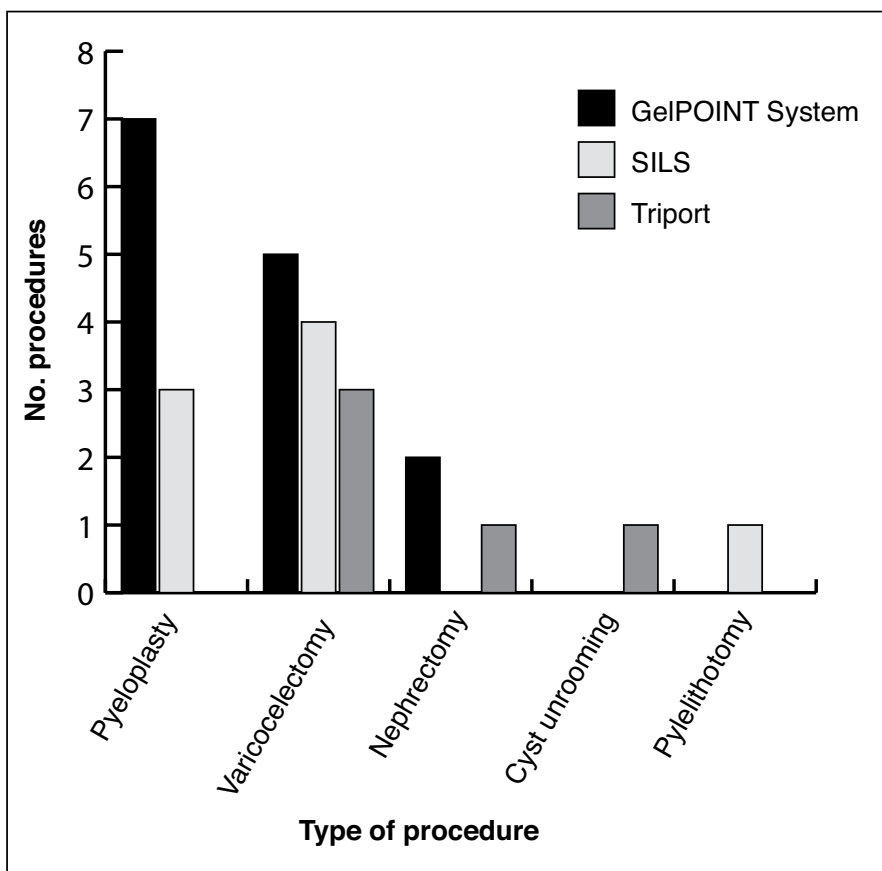

Fig. 2. Procedure breakdown by device used.

gical intervention. At 2 years postoperatively, the patient was normotensive and had not undergone any additional intervention. All patients who had a TL-P had favourable ultrasound findings postoperatively.

LESS varicocelectomy was performed in 5 patients, and all were discharged on the day of surgery. No complications were reported. The mean and median total operative times were 54 minutes and 55 minutes, respectively. The longest case was a unilateral case lasting 61 minutes. Similar to the pyeloplasty analysis, patients were compared to an agedmatched cohort of laparoscopic varicocelectomies. There was no difference in mean operative time (mean: 48 minutes, $p=0.4$ ). Resolution of varicoceles was seen in all cases at the postoperative follow-up ultrasound.

The LESS simple nephrectomies, LESS pyelolithotomy, and LESS renal cyst decortication were performed without complications or need for conversion. We did not perform an aged-matched cohort analysis due to the small number of these cases. Overall, all LESS cases were performed without the need for the insertion of an additional trocar.

\section{Discussion}

Laparoscopic surgery has the advantage of a smaller incision compared with open surgery, resulting in less postoperative pain, faster recovery, and improved cosmesis. ${ }^{8,9}$ The success of laparoscopic nephrectomy in pediatrics led to the adoption of this technique in management of various conditions, such as UPJ obstruction and intesex state. ${ }^{10,11}$ However, with each port insertion, there is a risk of visceral and vascular injury, and the potential of developing incisional hernias. To further reduce the morbidity of minimally-invasive surgery, techniques such as LESS are gaining more attention as an alternative to conventional laparoscopy.

LESS was first reported in 1998 for cholecystectomy and appendectomy. ${ }^{12,13}$ This technique uses a single skin and fascial incision for placement of ports. Advances in surgical techniques and instruments, such as multichannel singleaccess ports, novel articulating instruments and thin flexible laparoscopic cameras, have allowed the integration of LESS in urologic surgery. Transumbilical LESS is practical and affords a virtually scar-free approach. Another theoretical advantage includes reduced analgesic requirements, ${ }^{14}$ although this remains controversial due to the absence of randomized data in the pediatric population.

\begin{tabular}{|c|c|c|c|c|}
\hline Patient no. & Age (years) & Procedure & Surgical time (mins) & Length of stay (days) \\
\hline 1 & 10.0 & Cyst unroofing & 143 & 1.8 \\
\hline 2 & 10.0 & Simple nephrectomy & 153 & 1.5 \\
\hline 3 & 9.0 & Simple nephrectomy & 218 & 1.8 \\
\hline 4 & 14.0 & Pyelolithotomy & 277 & 1.6 \\
\hline 5 & 15.0 & Pyeloplasty & 233 & 1.5 \\
\hline 6 & 14.5 & Pyeloplasty & 155 & 1.8 \\
\hline 7 & 7.5 & Pyeloplasty & 244 & 2.5 \\
\hline 8 & 17.0 & Pyeloplasty & 219 & 1.5 \\
\hline 9 & 18.0 & Pyeloplasty & 250 & 2.5 \\
\hline 10 & 9.0 & Pyeloplasty & 244 & 2.5 \\
\hline 11 & 8.0 & Pyeloplasty & 218 & 2.6 \\
\hline 12 & 14.5 & Left varicocelectomy & 53 & 0 \\
\hline 13 & 15.0 & Left varicocelectomy & 61 & 0 \\
\hline 14 & 17.0 & Left varicocelectomy & 45 & 0 \\
\hline 15 & 13.0 & Bilateral varicocelectomy & 55 & 0 \\
\hline 16 & 17.5 & Left varicocelectomy & 55 & 0 \\
\hline
\end{tabular}


Complex reconstructive and extirpative urologic procedures have been performed using LESS. Although mostly in adults, these have included simple, partial, and radical nephrectomies, pyeloplasty, ileal ureter, and radical prostatectomy. ${ }^{15-17}$ There have been only a few retrospective case series looking at the feasibility and success of LESS in the urologic pediatric population. ${ }^{18-20}$ Moreover, there is no published data on LESS from Canada. In 2010, Koh and colleagues reported one of the first series of pediatric nephrectomies with LESS in 11 patients, with a mean age of 5.7 years. ${ }^{18}$ However, 5 early cases required insertion of an accessory port. Kocherov and colleagues published their results of LESS for a total of 14 procedures, including nephrectomy, gonadectomy, and varicocelectomy. They found no difference in the mean operative time, length of stay, and narcotic requirements with a similar cohort of patients who underwent conventional laparoscopy. ${ }^{19}$

In 2011, we presented the use of single incisional laparoscopic sugery (SILS) approach in a series of children who underwent 1- and 2-stage orchidopexy. ${ }^{21}$ This involved a single skin incision, but multiple fascial incisions through which the trocars were placed. The successful uptake of this technique led to the introduction of LESS for many urologic surgeries at our centre that were usually performed using the traditional laparoscopic approach. This gave us the opportunity to assess the outcomes and cost-effectiveness of this technique. As there is no standard approach to using the different available technologies for LESS, we alternated the use of different ports and instruments for each case to better assess their performance.

Our study supports the use of LESS in the pediatric population. Using multiple devices, we performed 5 different procedures, including pyeloplasty, varicocelectomy, nephrectomy, renal cyst decortication, and pyelolithotomy via an umbilical incision resulting in a concealed surgical scar. In only 1 case a pyeloplasty required conversion to open due to a small intra-renal pelvis. None of these cases required insertion of an additional 5-mm port or a 3-mm percutaneous instrument. There were no differences in hospital length of stay and operative times of the LESS procedures when compared to a similar cohort of conventional laparoscopic cases. All patients with varicocelectomies were discharged the same day, and the hospital length of stay of about 2 days for the LESS-P group was either comparable or better than the earlier series. ${ }^{22,23}$ The cost reported for LESS-P was based on the cost of a heterogeneous group of port devices, ranging from $\$ 350$ to $\$ 650$ excluding the instruments. The reduced cost associated with LESS-pyeloplasty is unlikely to represent a true saving as the analysis was skewed due to a prolonged hospital stay of 1 patient in the TL-P group.

We consider that a universally beneficial new surgical approach needs to be easily learned and reproducible by other surgeons. Like other groups, we found that LESS requires more advanced techniques compared with multiport laparoscopic surgery because the instruments are introduced adjacent and parallel to each other through a single port and the surgeon has a limited range of motion. We currently offer LESS for specific procedures, such as varicocelectomy, nephrectomy, and pyeloplasty. We traditionally use either the SILS or Gelpoint technologies with a combination of articulating and bent instruments to partially overcome the loss of triangulation and "clashing" of instruments. Finally, although the learning curve with LESS has not been robustly explored, proficiency may require an advanced laparoscopic skillset, especially for intracorporeal suturing.

Given that there are only a few retrospective studies, our initial goal was to explore the utility of the LESS technique for urologic procedures, and the results support earlier observations of this being a viable option in children. Unlike most of the earlier pediatric series, we also performed a matched cohort analysis with patients who underwent traditional laparoscopy for pyeloplasty and varicocelectomy, and found no significant difference between the 2 techniques. Although this represents a limited series, our study is the first Canadian experience with LESS, and contributes to the growing evidence supporting the use of this technique.

However, our study has several limitations. The series included a heterogeneous patient population who underwent different procedures with a short follow-up period. Moreover, all surgeries were performed by the same surgeon (WF), with a vast experience in pediatric laparoscopy, at a tertiary centre. Hence, the generalizability of these results cannot be assessed at this stage. Ultimately, randomized trials are required to assess for LESS's superiority over conventional laparoscopy in terms of narcotic requirements, length of hospital stay, financial cost, and patient satisfaction.

\section{Conclusion}

LESS offers the opportunity to perform laparoscopic abdominal procedures in a patient using a single concealed incision within the umbilicus. Although our experience is still limited, the present cohort demonstrates the safety and feasibility of transumbilical LESS in the pediatric urological population. Randomized studies are required to better define the role of LESS.

Competing interests: Authors declare no competing financial or personal interests.

This paper has been peer-reviewed. 
Khambati et al.

\section{References}

1. Kaouk J, Haber G, Goel R, et al. Single-port laparoscopic surgery in urology: Initial experience. Urology 2008;71:3-6. http://dx.doi.org/10.1016/j.urology.2007.11.034

2. Raman J, Bensalah K, Bagrodia A, et al. Laboratory and clinical development of single keyhole umbilical nephrectomy. Urology 2007;70:1039-42. http://dx.doi.org/10.1016/j.urology.2007.10.001

3. Rane A, Rao P, Rao P. Single-port-access nephrectomy and other laparoscopic urologic procedures using a novel laparoscopic port (R-port). Urology 2008;72:260-3. http://dx.doi.org/10.1016/j.urology.2008.01.078

4. White W, Haber G, Goel R, et al. Single-port urological surgery: Single-center experience with the first 100 cases. Urology 2009;74:801-4. http://dx.doi.org/10.1016/j.urology.2009.04.030

5. Kaouk J, Palmer J. Single-port laparoscopic surgery: Initial experience in children for varicocelectomy. BJU Int 2008;102:97-9. http://dx.doi.org/10.1111/j.1464-410X.2008.07584.x

6. Aboutkidshealth.ca. Hospital for Sick Children Online Resources; c2004-14 [updated 2010 March 9]. http://bit.ly/1Bf322Y. Accessed January 26, 2015.

7. Wehbi E, Kanaroglou N, Farhat W. Laparoendoscopic single-site pyelolithotomy in a pediatric patient. J Endourol, Videourology 2013;27. http://dx.doi.org/10.1089/vid.2013.0069

8. Borin J. Laparoscopic radical nephrectomy: Long-term outcomes. Curr Opin Urol 2008;18:139-44. http:// dx.doi.org/10.1097/MOU.0b013e3282f4a851

9. Eskicorapci S, Teber D, Schulze, et al. Laparoscopic radical nephrectomy: The new gold standard surgical treatment for localized renal cell carcinoma. ScientificWorldJournal 2007;7:825-36. http://dx.doi. org/10.1100/tsw.2007.153

10. Koyle M, Woo H, Kavoussi L. Laparoscopic nephrectomy in the first year of life. J Pediatr Urol 1993;28:6935. http://dx.doi.org/10.1016/0022-3468(93)90034-I

11. Chertin B, Koulikov D, Alberton J, et al. The use of laparoscopy in intersex patients. Pediatr Surg Inter 2006;22:405-8. http://dx.doi.org/10.1007/s00383-006-1662-3

12. Piskun $G$, Rajpal $S$. Transumbilical laparoscopic cholecystectomy utilizes no incisions outside the umbilicus. J Laparoendosc Adv Surg Tech A 1999;9:361-4.

13. Esposito C. One-trocar appendectomy in pediatric surgery. Surg Endosc 1998;12:177-8.

14. Autorino R, Brandao L, Sankari B, et al. Laparoendscopic single-site (LESS) vs laparoscopic living-donor nephrectomy: A systematic review and meta-analysis. BJU Int 2015;115:206-15. Epub 2014 July 15. http://dx.doi.org/10.1111/bju.12724
15. Desai $M$, Aron $M$, Rao $P$, et al. Scarless single port transumbilical advanced laparoscopic surgery: Initial report of nephrectomy, pyeloplasty and partial nephrectomy. J Urol 2008;179:239. http://dx.doi. org/10.1016/S0022-5347(08)60688-6

16. Desai $M$, Stein R, Rao P, et al. Embryonic natural orifice transumbilical endoscopic (E-NOTES) for advanced reconstruction: Initial experience. Urology 2009;73:182-7. http://dx.doi.org/10.1016/i. urology.2008.04.06

17. Kaouk J, Goel R, Haber G, et al. Single-port laparoscopic radical prostatectomy. Urology 2008;72:1190-3. http://dx.doi.org/10.1016/i.urology.2008.06.010

18. Koh C, De Filippo R, Chang A, et al. Laparoendoscopic single-site nephrectomy in pediatric patients: Initial clinical series of infants to adolescents. Urology 2010;76:1457-61. http://dx.doi.org/10.1016/i. urology.2010.06.066

19. Kocherov $S$, Lev $G$, Shenfeld 0 , et al. Laparoscopic single site surgery: Initial experience and description of techniques in the pediatric population. J Urol 2011;186:1653-7. http://dx.doi.org/10.1016/i. juro.2011.03.100

20. Bansal D, Cost N, Bean C, et al. Pediatric urologic laparoendoscopic single site surgery: Single surgeon experience. J Pediatr Urol 2014;10:1170-5. Epub 2014 June 19. http://dx.doi.org/10.1016/j. ipurol.2014.04.020

21. El-Hout $Y$, Romao R, Weber B, et al. Single incision laparoscopic surgery (SILS) in children: Initial experience. AUA University May 2011; V1707. http://bitly/1EMyyc2. Accessed January 26, 2015.

22. Tugcu $\mathrm{V}$, llbey $\mathrm{Y}$, Polat $\mathrm{H}$, et al. Early experience with laparoendoscopic single-site pyeloplasty in children. J Pediatr Urol 2011;7:187-91. http://dx.doi.org/10.1016/i.jpurol.2010.10.014

23. Zhou H, Sun $\mathrm{N}$, Zhang $\mathrm{X}$, et al. Transumbilical laparoendoscopic single-site pyeloplasty in infants and children: Initial experience and short-term outcome. Pediatr Surg Int 2012;28:321-5. http://dx.doi. org/10.1007/s00383-011-3040-z

Correspondence: Dr. Walid A. Farhat, The Hospital for Sick Children, University of Toronto, Toronto, ON; walid.farhat@sickkids.ca 